\title{
Editorial
}

\section{Insights into the regulatory mechanisms in the cellular protein processing factory}

\author{
$J$ Yuan $^{*, 1}$ \\ ${ }^{1}$ Department of Cell Biology, Harvard Medical School, Boston, MA, USA \\ * Corresponding author: J Yuan, Department of Cell Biology, Harvard Medical \\ School, 240 Longwood Ave, Boston, MA 02115, USA. Tel: 617432 4170; \\ Fax: 617432 4177; E-mail: jyuan@hms.harvard.edu
}

Cell Death and Differentiation (2006) 13, 359.

doi:10.1038/sj.cdd.4401842

The endoplasmic reticulum (ER) serves as the primary cellular protein processing factory where proteins destined for secretion or membrane insertion are processed, modified and folded. This membrane-bound organelle recruits translating ribosomes, translocates newly synthesized peptides into its lumen, and promotes a variety of post-translational modifications and chaperone-facilitated folding events. Proper ER function is therefore critical for numerous aspects of cell physiology, including vesicle trafficking, lipid and membrane biogenesis and protein targeting and secretion. Perturbation of ER function leads to rapid accumulation of unfolded and misfolded proteins. Metazoan cells react rapidly to ER dysfunction through a set of adaptive pathways known collectively as the ER stress response (ESR). The ESR pathways function in a manner analogous to when we try to get rid of excess junk from our houses by getting additional cleaning workers (synthesizing additional chaperone proteins), stopping buying more junk (inhibition of protein synthesis) and throwing away junk (ER-associated protein degradation). Prolonged ER dysfunction, however, may lead to something that we would not do when we accumulate too much junk: to commit cellular suicide (apoptosis). The pathways of the ESR were illustrated by elegant genetics in budding yeast, molecular genetic analysis of analogous pathways in mammals and recently by chemical biology (reviewed in Boyce and Yuan ${ }^{1}$ and Wu and Kaufman ${ }^{2}$ ).

The ESR is an interesting cellular mechanism that is most likely evolved as a physiological response involved in regulating normal physiological processes and then adapted to function under pathological conditions. In secretory cells, such as plasma cells, pancreatic $\beta$ cells and hepatocytes, the ESR serves as an indispensable physiological regulator in maintaining homeostatic balance of signal-input-output. ${ }^{2}$ On the other hand, in most nonsecretory cells, the ESR functions under pathological conditions to ameliorate unfolded protein overload in the ER and to mediate cell death under extreme conditions. $^{3}$

The regulatory mechanisms of the ESR are extensively involved in multitude of other cellular processes including cell differentiation, glucose metabolism and cellular defense mechanisms. As an example, phosphorylation of elF $2 \alpha$, which leads to inhibition of protein synthesis during ER stress, also occurs prominently during interferon $\gamma$ stimulation and viral infection, ${ }^{4}$ and, thus, provides a crosstalk point between the ESR and other cellular processes such as cytokine and inflammatory signaling pathways, antiviral responses and cell death.

Intracellular mediators of ESR include not only proteins, ions such as calcium, but also gangliosides, a subclass of glycosphingolipids and an important intracellular regulator of membrane dynamics. ${ }^{5}$ The ability of gangliosides to integrate signaling mechanisms in the membrane compartments of different subcellular organelles, including ER, Golgi, mitochondria and lysosomes, etc., provides a new lipid dimension for ESR.

Given the extensive and intricate connection of ER function with multiple other cellular processes, it is not surprising that ER stress is implicated in a multitude of human diseases including diabetes, chronic and acute neurodegenerative disorders. ${ }^{1-5}$ I sincerely hope this collection of reviews will serve to guide and inspire new researches in this area and to lead to the development of new treatments for human diseases.

1. Boyce M and Yuan J (2006) Cell Death Differ. 13: 363-373

2. Wu J and Kaufman RJ (2006) Cell Death Differ. 13: 374-384

3. Lindholm D, Wootz H and Korhonen L (2006) Cell Death Differ. 13: 385-392

4. He B (2006) Cell Death Differ. 13: 393-403

5. d'Azzo A, Tessitore A and Sano R (2006) Cell Death Differ. 13: 404-414 